\title{
Visual word recognition of three-letter words as derived from the recognition of the constituent letters
}

\author{
DON BOUWHUIS and HERMAN BOUMA \\ Institute for Perception Research, IPO, P.O. Box 513, 5600 MB Eindhoven, The Netherlands
}

\begin{abstract}
Word recognition is one of the basic processes involved in reading. In this connection, a model for word recognition is proposed consisting of a perceptual and a decision stage. It is supposed that, in the perceptual stage, the formation of possible words proceeds by separate identification of each of the letters of the stimulus word in their positions. Letter perception is taken to be conditional on position because of interaction effects from neighboring letters. These effects are dependent on both position in the word and retinal eccentricity, which are of particular relevance in reading. The letter-based approach rests on the strong relationship between the results from single-letter recognition in meaningless strings and in real words. Next, in the decision step, the many alternatives generated in the perceptual stage are matched with a vocabulary of real words. It is supposed that the final choice from among the remaining words is made in accordance with the constant ratio rule; frequency effects are not separately incorporated in the model. All predictions of the model are generated by means of data from earlier experiments. Despite being not optimally suited for this purpose, the predictions compare favorably with responses in word-recognition experiments.
\end{abstract}

In this article, a model is reported which describes the recognition of single words of three letters on the basis of the perception of the constituent letters. The model explains more fully the results of an earlier, extensive experiment on the recognition of letters and words carried out by Bouma (1973), which was part of a general investigation of visual processes involved in normal reading. In that experiment, an attempt was made to determine which attributes of words contribute to word recognition, particular attention being paid to explaining the role of initial and final letters of words. In addition, the experiment studied how the perception of a letter in a word related to that of the same letter in a meaningless string of the same length.

Full implementation of the model made it necessary to run a supplementary experiment on the recognition of middle letters in meaningless strings. Having already obtained data on initial- and final-letter recognition (Bouma, 1973), quantitative predictions could then be attempted for words consisting of three letters. The experiments were restricted to parafoveally presented words. Under reading-like situations, fixated words can always be correctly perceived and consequently yield no information on the attributes mediating their perception. Since all words in a text shift under the reader's eye, all are at one or more times parafoveal.

We owe many thanks to Mr. A. L. M. van Rens who collected the data in the experiments, to Mr. J. C. Jacobs for participating in the tedious phase of the model testing, and to Dr. C. Schiepers, who assisted in the compilation of the word vocabulary.
The manner in which the perception of letters operates in word recognition, according to the model, will be described in the following section. Other proposals, notably those by Morton (1969) and by Rumelhart and Siple (1974), will be discussed in connection with the present formulation. Next, the original recognition experiments will be summarized in order to clarify what the model is intended for and how the parameters are obtained.

The model was tested on the results of a wordrecognition experiment (Bouma, 1973) in which 11 subjects participated. Three types of analysis are presented, each involving different aspects of the response words. Finally, the implication of the good fit of the model to the experimental data will be discussed in connection with the role of global word shape, word frequency, and word knowledge.

\section{THE LETTER CONFUSION MODEL FOR WORD RECOGNITION}

It is assumed here that when a word of three letters is presented, the three constituent letters in their particular positions are usually perceived imperfectly or sometimes hardly at all. Which letters will then be seen is taken to be dependent on the position of the presented letters and on their characteristic features.

This view implies the notion that position-specific properties include the main perceptual dependence (interference) between neighboring letters. Since letter perception is a probabilistic process, at least under 
conditions of parafoveal presentation, a combination rule for the probability of a specific combination of letters must be given.

The most straightforward combination rule for this case is that the letters of the perceived letter string are generated independently of the perception of other letters. The probability that one particular letter string will be perceived is then the product of the three separate probabilities for each letter. Thus, each of the latter probabilities is dependent on the letter which was actually contained in the presented word and on the position it took in the word. Mutual interference is therefore taken to be positionspecific. For example, with the stimulus word "wet" (law) the string "vat" (barrel) would result if the observer had perceived a " $v$ " for the initial " $w$," an " $a$ " in the middle for the " $e$," and the letter " $t$," correctly at the end. This process of string activation is indicated in the upper part of Figure 1 showing the computer output of the model test. The probability that the letter $w$ will be perceived as " $v$ " is designated here as $P(v--\mid w--)$. The probability that the whole string "wet" will be perceived as the string "vat" can then be written as:

$$
P(\text { vat } \mid \text { wet })=P(v--\mid w--) \cdot P(-a-\mid-e-) \cdot P(--t \mid--t) \text {. }
$$

Thus, any stimulus word may activate a large number of strings, some of which will be real words; possible candidates for "wet" are "wel" (well), "wct" (-), etc. Real words, however, are generally far outnumbered by meaningless and often unpronounceable strings. But it is well known that subjects trying to recognize words tend to give real word responses even if sensory information is too scanty for correct identification. For words of three letters, nonword responses amounted to $2.5 \%$ in the Bouma experiment (1973). While the subject is at liberty to select a response from a mixed set of many meaningless strings and a few words, he seems to consider almost only existing words.

For the decision stage of the model, it is therefore assumed here that all meaningless strings are discarded from the response alternatives. This effectively agrees with experimental findings and has the added advantage that no selection parameter is needed, which would otherwise have to be estimated.

One suitable decision rule for the choice of the final word response is the constant ratio rule (Clarke, 1957). Let us denote a particular perceived letter string forming the word $i$ as $r_{i}$. The probability that this string will arise, according to the rule defined in Formula 1 , is then $p\left(r_{i}\right)$. The constant ratio rule then defines the following probability:

$$
Q\left(r_{i} \mid s_{j}\right)=\frac{p\left(r_{i}\right)}{\sum_{k} p\left(r_{k}\right)},
$$

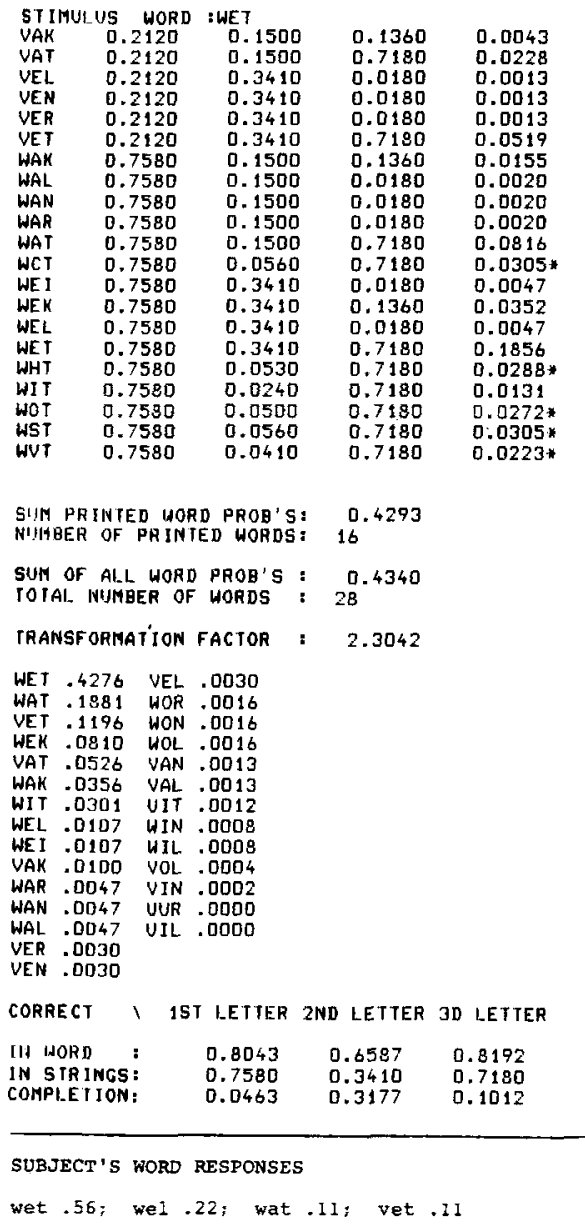

Figure 1. A sample of computer output of the model test for the word "wet" (law) presented at $-1.75^{\circ}$ visual angle. The first three columns give the probabilities that the first, second, and third letter, respectively, of the printed string were seen for the corresponding letters of the stimulus. The fourth column gives the product of the three probabilities. An asterisk denotes that the string is not a word. The transformation factor is the inverse of the sum of the word probabilities; multiplying the activation probabilities of the words with this factor is equivalent to the application of the constant ratio rule. Final responses with their predicted probabilities are shown in the bottom two columns. Correct letter probability in predicted words are shown in the lower table. The second row gives the experimentally obtained recognition scores of the same letters in meaningless strings. The difference between these is completion. The bottom row presents the responses of the subjects for the word "wet.",

where $s_{j}$ denotes the stimulus word and $r_{k}$ all activated words. These operations of the model are further exemplified for the word "wet" in Figure 1.

The constant ratio rule has the property that the activation probability of the whole letter string, which may be quite low, is transformed into a word response probability which is substantially higher, depending on the probability of alternative word responses. In general, the probability of correct word perception is so high that the probability of correct letters in the predicted responses exceeds the probability of correct letter perception in meaningless 
strings. This phenomenon is found in experiments where subjects may infer from their knowledge of a word the letters of that word which they have not perceived well. Bouma (1973) called this effect completion, implying that the subjects complete the partially seen word. The same effect has also been termed word superiority effect (Wheeler, 1970) appearing in more or less differing experimental paradigms. The effect goes as far back as Cattell, cited by Woodworth (1938), in which an excellent historical review can be found. The application of the constant ratio rule also makes it clear that no effects of differential word frequency are accounted for in the present version of the model. Only visual factors and word knowledge are taken to be operative in word recognition under the conditions studied.

\section{ALTERNATIVE MODELS}

Recently proposed models for visual word recognition include Morton's logogen model (1969) and the multicomponent model put forward by Rumelhart and Siple (1974). The logogen model does not contain a specification of word or letter confusion but the multicomponent model does. Both the latter and the present letter confusion theory can predict responses for arbitrary words.

\section{The Logogen System}

The basic unit of Morton's model (1969) is the logogen, which corresponds to a word in the language. During visual processing of stimulus words, the logogen is fed information from two sources, the visual system, providing sensory information, and the context system, which raises expectations for particular words. The effects of sensory information, $\alpha$, and context information, $\beta$, combine independently, in a multiplicative way, to form the response strength, $\alpha \beta$, for a given word. During reading, the response strength is dependent on context, which causes variability in the factor $\beta$ and consequently in the response strength. Predictions of responses are made in the framework of choice theory (Luce, 1959). Specifically, the probability of a response, $r_{i}$, is equal to its strength, $(\alpha \beta)_{\mathrm{i}}$, divided by the sum of all other response strengths:

$$
\mathrm{Q}\left(\mathrm{r}_{\mathrm{i}} \mid \mathrm{s}_{\mathrm{j}}\right)=\frac{(\alpha \beta)_{\mathrm{i}}}{\sum_{\mathrm{k}}(\alpha \beta)_{\mathrm{k}}},
$$

which is equivalent again to the constant ratio rule. Formula 3 is similar to Formula 2, defining the decision rule for the letter confusion model. But the terms $(\alpha \beta)_{k}$ also show a formal correspondence with the present model.

In experiments concerned with the recognition of single, unconnected words, the influence of the con- text system is minimized. In such a case, the stimulus and the visual system are the main sources of variability in the response strengths, allowing the value of $\beta$ to be set to 1 . Now, in Formula 1, recognition information from the constituent letters is integrated by way of multiplication too. Its formal counterpart in the logogen model would be $\left(\alpha_{1} \alpha_{2} \alpha_{3}\right)_{k}$, the subscript denoting the three letters, whereas the only difference compared with Formula 1 is that the response strength is only unique up to multiplication by a positive constant. But, though the equations are formally identical, the interpretation differs for the two models. The response strength, $\alpha$, is originally defined for the whole stimulus word and not for the constituent letters. In the present proposal, measurements on the separate letters represent a kind of sensory interpretation of the $\alpha$ factor within the framework of the logogen model.

The original version of the logogen model is rather more detailed with respect to availability of responses. There is generally a parameter, $V$, combined with the response strength $(\alpha)_{\mathrm{k}}$, like $(\alpha \mathrm{V})_{\mathrm{k}}$, reflecting the effect of word frequency.

Tests of the logogen model have mainly centered on the availability effects and influence of context (Morton, 1969). Disregarding differences in availability, it should finally be noted that logogens, by definition, correspond to real words only. In this way, selection of real words from possibly activated strings as is required in the letter confusion model, is automatically accomplished.

\section{The Multicomponent Model}

The word-recognition model proposed by Rumelhart and Siple (1974) encompasses processes responsible for the development of a sensory image of the stimulus word up to the final production of the response word. Here, only the stimulus description and the decision rule will be briefly discussed.

Letters constituting the stimulus words employed by Rumelhart and Siple (1974) consist of straightline segments, which they call "functional features." The probability of detection, $t_{i}$, of any one feature, $f_{i}$, is assumed to be independent of the presence or absence of other features. For a particular letter consisting of four features, only the features $f_{1}$ and $\mathrm{f}_{3}$ may be detected in a given trial. The probability $\mathrm{q}$ of this subset arising is then given by:

$$
q=P\left(f_{1}, \bar{f}_{2}, f_{3}, \bar{f}_{4} \mid l\right)=t_{1}\left(l-t_{2}\right) t_{3}\left(l-t_{4}\right),
$$

where 1 denotes the letter in the word. Letter confusion is introduced here since the two detected features might also belong to other letters. At this stage, it is not letters that are mediating agents for the response but parts of letters. A consequence of independence of detection is that the probability of detecting feature sets for all three letter positions is: 


$$
P\left[F\left(l_{1}\right), F\left(l_{2}\right), F\left(l_{3}\right) \mid s_{j}\right]=\left(q_{1} q_{2} q_{3}\right)_{j},
$$

where $F\left(l_{\mathfrak{i}}\right)$ denotes the set of extracted features from the letter in position $i$ in the word $s_{j}$. These sets of features, $F$, might also have been extracted from other letters in other words. The probability of these would generally be different, since other letters contain either more or fewer features and, in any case, different features. The final response probability is defined as in the former cases:

$$
\mathrm{Q}\left[\mathrm{r}_{\mathrm{i}} \mid \mathrm{F}\left(\mathrm{l}_{\mathrm{l}}\right), \mathrm{F}\left(\mathrm{l}_{2}\right), \mathrm{F}\left(\mathrm{l}_{3}\right)\right]=\frac{\left(\mathrm{q}_{1} \mathrm{q}_{2} \mathrm{q}_{3}\right)_{\mathrm{i}} \mathrm{b}_{\mathrm{i}}}{\sum_{\mathrm{k}}\left(\mathrm{q}_{1} \mathrm{q}_{2} \mathrm{q}_{3}\right)_{\mathrm{k}} \mathrm{b}_{\mathrm{k}}},
$$

where $b_{k}$ is the subject's a priori probability that stimulus $s_{k}$ will be presented. This may be conceived as analogous to the factor $\beta$ in the logogen model. If this factor is disregarded here for the same reasons as before, Formulas 5 and 6 again show a formal correspondence with Formulas 1 and 2. However, since Formula 6 is defined for parts of letters, the predictions of the multicomponent model employ Formula 6 for all different feature sets which may be extracted from the three letters of one stimulus word. The difference compared with the present model of letter confusion to be noted here is the elementary assumption of Rumelhart and Siple (1974) that letter or feature position does not influence detectability. Though this might have been applicable in the experimental conditions employed by Rumelhart and Siple (1974), it is not valid in general, certainly not for eccentric word presentation.

Frequency effects enter into the picture in a complicated way, since Rumelhart and Siple (1974) predict responses consisting not only of real words, but of syllables and meaningless strings as well. In a simulation of the model, Rumelhart and Siple (1974) tried to account for all responses to 726 words and strings of three letters with known frequencies in the printed language. Predictions of the theory, making use of approximations of the parameters, appear to be representative of gross effects. Frequency effects figure predominantly in the data analysis. Letter confusability, which can easily be derived from the multicomponent theory and which is a purely visual effect, produces variations in recognition probability that are much larger than those produced by differences in word frequency.

\section{A TEST OF THE LETTER CONFUSION MODEL}

The recognition model was tested on the results of the word recognition study by Bouma (1973); for the letter confusion predictions, the letter recognition data obtained in the study were utilized. In that experiment, words consisting of $3,4,5$, and 6 letters were pre- sented in random order to 11 subjects. The initial and final letters of these words had been presented in an earlier phase, appearing in the same position in meaningless strings. These meaningless strings were derived from the original words by replacing all letters except the initial and final ones by perceptually similar letters, leaving the word contour intact. Similarity had been established by Bouma (1971) in an experiment involving single-letter recognition. Table 1 shows the similarity groups from which the replaced letters were selected and the way in which the words were transformed into meaningless strings. In these strings, the observer can no longer infer the letter asked for from his knowledge of the word, whereas interaction effects are assumed to be similar. Letter recognitions and confusions in this situation, therefore, may reflect the real contributions of letters in the perception of words.

For the recognition data of the middle letters of words of three letters, a supplementary experiment was run using only the three-letter words to be transformed into strings, according to the scheme shown in Table 1.

The experimental details have been published extensively elsewhere (Bouma, 1973) and will only be repeated here for a few relevant details.

\section{Method}

Stimuli. Word stimuli were well-known Dutch words with frequencies of occurrence between $10^{-6}$ and $10^{-3}$ in printed Dutch. The model was tested on a subset of the presented words, namely 100 three-letter words presented once-left and once right of the fovea for all subjects. The eccentricities were $\pm 1.75^{\circ}$ and $\pm 2.75^{\circ}$ of visual angle, referring to the letter closest to the fovea. For other eccentricities, too few observations had been made to include them in the test of the model.

Letter recognition trials followed the rationale of the word recognition study. Subjects reported both the initial and final letter of the presented unpronounceable letter string. In the later supplementary experiment, designed for a test of the model, subjects reported only the middle letter. Thus, confusion matrices for three letter positions in four eccentricities were obtained.

Subjects. Eleven subjects with (corrected) vision above 1.0 participated in the experiments. Most of them were experienced observers but had no prior information as to the exact purpose of the present experiment. The same 11 subjects participated in the later experiment on the middle letters.

Table 1

The Seven Similarity Groups of Letters Employed in the Transformation of Words Into Unpronounceable Letter Strings for the Study of Recognition of

\begin{tabular}{|c|c|c|c|}
\hline \multicolumn{4}{|c|}{ Transformation } \\
\hline Words & String & Repo & \\
\hline $\begin{array}{l}\text { gas } \\
\text { lip }\end{array}$ & $\begin{array}{l}\text { gzs } \\
\text { lfp }\end{array}$ & \multicolumn{2}{|c|}{ Initial and Final Letters } \\
\hline $\begin{array}{l}\text { arm } \\
\text { fee }\end{array}$ & $\begin{array}{l}\text { sru } \\
\text { ieo }\end{array}$ & \multicolumn{2}{|l|}{ Middle Letters } \\
\hline \multicolumn{4}{|c|}{ Similarity Groups } \\
\hline \multicolumn{2}{|c|}{ Short Letters } & Ascenders & Descenders \\
\hline a $s z \times$ e oc & $u r v w$ & $\mathrm{t}$ i l f $\mathrm{d} \mathrm{h} \mathrm{k} \mathrm{b}$ & $\mathrm{g} p$ j y $q$ \\
\hline
\end{tabular}
Constituent Letters 
Apparatus. Strings and words were presented by means of a two-channel tachistoscope with field dimensions of $30 \times 30 \mathrm{~cm}$. Stimuli were typed in lowercase Courier 10 typeface with carbon tape. The height of a short letter subtended a visual angle of $.20^{\circ}$, with $.28^{\circ}$ for ascenders and descenders. One letter space corresponded to $.25^{\circ}$ visual angle. The subjects fixated on a " $+"$ in the middle of the rest field, which was replaced, for $100 \mathrm{msec}$, by the eccentric stimulus word. This duration was chosen to avoid the influence of inadvertent eye movements which might occur in longer durations.

Word lists. Next to letter recognition data, the model requires a word vocabulary in order to select the words from among the letter strings perceived for the presented words. This vocabulary should then match the subject's word knowledge as closely as possible. In the first testing phase of the model, there was only one representative count of Dutch words, that of De la Court (Linischoten, Note 1) which dated from 1937 and comprised $1,000,000$ words. In 1975, however, a new count of 720,000 words of printed Dutch was published by Uit den Boogaart (1975). In this count, a word was conceived as anything between two spaces. A first list of three-letter words was compiled from this count by discarding proper names, designations, abbreviations, and numbers. Thus, 409 words were obtained with which a first test of the model was run. When it was discovered that this list was incomplete, it was extended with supplementary words from the De la Court count (Linschoten, Note 1) and with the results of questionnaires handed out to some readers in order to ensure that adopted words were known. Frequencies of occurrence were known for the words appearing in the counts, but not employed in the predictions. Total number of words in the extended list was 541 .

\section{A Comparison of Predictions and Experimental Results}

For purposes of clarity, a computer printout of the model test is shown in Figure 1 for the word "wet" (law) presented at $-1.75^{\circ}$ visual angle. At the bottom of the figure, are shown corresponding experimentally obtained responses against which the predictions were tested. For all stimulus words in the experiment, the probability of recognition responses could only roughly be established because of the limited number of subjects, resulting in only 11 responses per stimulus word. Since longer words had also been present in the original experiment, some of the error responses to three-letter words contained two, four, or five letters. Finally, some nonsense words and responses of "illegible" were obtained. Together with the different-length responses, they amounted to $11 \%$ of all responses.

By definition, the model is limited to the prediction of only real words of three letters, and a comparison should therefore be based on the appropriate subset of responses. The effect of leaving these nonpredicted responses out of account was to increase

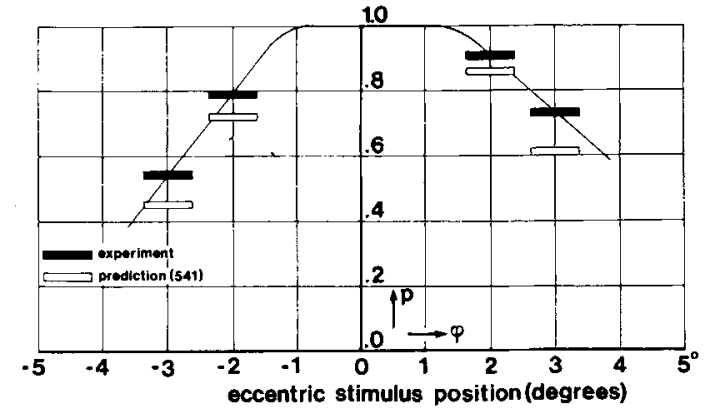

Figure 2. Average correct scores for $\mathbf{5 0}$ different words as a function of eccentricity. Bars indicate the extent of one word of three letters.

the correct experimental scores by .06 on average and the incorrect scores by somewhat less. The assumption behind this decision was that, by normalizing all relative response frequencies, the same responses are obtained as would show up if the subjects had reported only real-word responses of three letters. Here only those predictions are reported that are based on the 541-word vocabulary.

Correct word scores. The correct word scores, pooled over all stimulus words, are shown in Table 2, both predictions and experimental values. Again, it is stressed here that the predictions are based on letter-recognition data from a separate experiment. Even for one eccentricity, stimulus words vary considerably in recognition probability. This variation is expressed as the standard deviation of the correct scores, also shown in Table 2 . The agreement between experimental correct scores and predictions is shown in Figure 2. A consistent underestimation of the correct scores by the predictions is to be noted, amounting on average to .08 . A smaller underestimation appears in the standard deviations.

A typical picture of the relation between predictions and experimental values is shown in Figure 3. With respect to the reliability of the experimental data, it should be noted that, for example, for an average of 8 correct responses out of $11(73 \%)$ one standard deviation is $1.5(13 \%)$. Though the correlation coefficient is less appropriate for probabilities, it amounts to 0.66 in Figure 3.

Letter scores. A more sensitive test of the predictions can be made on the basis of correct letters in the responses. Correct letters appear not only in the correct responses, but also in most error re-

Table 2

Average Correct Scores and Their Standard Deviations as a Function of Eccentricity of Presentation

\begin{tabular}{lrrrrrrrr}
\hline & \multicolumn{7}{c}{ Eccentricity (in Degrees) } \\
\cline { 2 - 8 } & \multicolumn{3}{c}{-2.75} & & -1.75 & +1.75 & +2.75 \\
\hline Average Correct Score & .544 & $(.472)$ & .791 & $(.720)$ & .904 & $(.858)$ & .733 & $(.607)$ \\
Standard Deviation & .234 & $(.185)$ & .215 & $(.210)$ & .138 & $(.126)$ & .206 & $(.189)$ \\
\hline
\end{tabular}

Note-There were 50 words per eccentricity. Predictions by the model are shown in parentheses. 


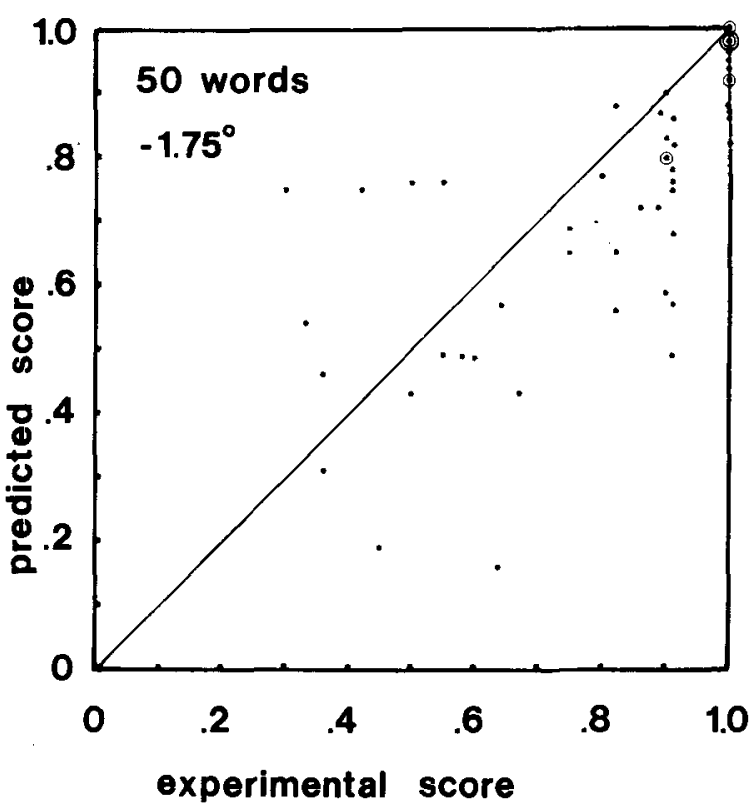

Figure 3. Observed and predicted correct scores for 50 different words presented at $1.75^{\circ}$ visual angle left of the fovea.

sponses. According to the basic assumption of the model, they are only considered to be correct if they are in their proper position in the word. Part of the letter recognition is contributed by completion. The completion score is defined as the difference in probabilities that a letter in a word response is correct and that it is correctly reported in a meaningless string. Figure 4 shows the predicted and observed correct letter scores in predicted responses as well as the letter recognition scores for all letter positions as a function of eccentricity. There is still a slight underestimation, amounting to less than .02 on average.

Distributions of experimental and predicted responses. Many possible incorrect responses may be expected to have a low probability of occurrence. Even if substantially more subjects were to report words, these probabilities cannot satisfactorily be established. The next analysis, instead of comparing predicted and observed response probabilities, examines whether observed responses are likely to have originated from a response population as predicted by the model. Responses that are highly probable according to the model should also be frequent in the observed responses, whereas responses predicted to be improbable should hardly occur.

This correspondence was checked for each stimulus word separately in successive classes of predicted probability. To this end, the probability interval was partitioned in successive intervals, ranging from highly probable to very improbable. Each interval. boundary was chosen to be a factor of $\sqrt{.1}$ smaller than the preceding one. This choice led to eight probability classes, the highest of which is crude but which usually contains only correct responses, whereas the smaller ones become progressively more discriminating.

For each stimulus word, a number of responses are predicted by the model to fall in each of the probability classes. The number of response words produced by the subjects which matched the predicted words in one interval was scored and compared with the number of predicted responses.

The predicted numbers are plotted against the observed numbers in the respective probability intervals in Figure 5 for each stimulus eccentricity. Proportionality shows up nicely in a slope of almost 1.0. Observed numbers in the highest class, comprising almost exclusively correct responses, are again underestimated by the model in this analysis. The increasing scatter towards the lower left part of the plot is due to the inherent unreliability of small observed numbers. Of all observed responses, $7 \%$ were pre-

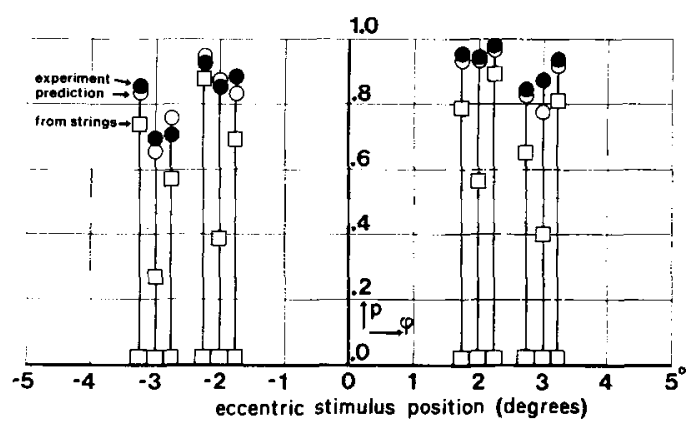

Figure 4. Comparison of letter recognition in words and strings at four eccentric positions. Squares indicate scores in strings, black dots, scores in words. Open circles are predictions of letter scores in words. The difference between scores in words and strings is called completion.

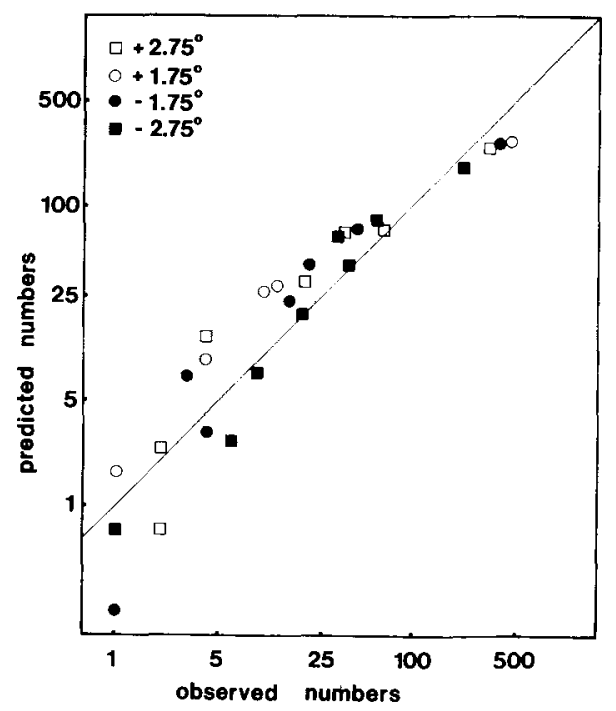

Figure 5. Observed and predicted numbers of responses for the four sets of words at four eccentricities. Each point refers to a particular probability interval, as explained in the text. 
dicted to have a probability of occurrence of $10^{-4}$ or less and were thus wrongly rejected by the model.

\section{GENERAL DISCUSSION}

The results of the test of the letter confusion model of letter recognition indicate that even this simple model can account for a great deal of the experimental data obtained in the word recognition experiments. This finding stresses the importance of the constituent letters in the perception of words, but, of course, does not prove that letters are the ultimate features for word perception. Letters were chosen in the present context for experimental simplicity, being easy to manipulate as identifiable units.

Parameters of the model were not estimated from word recognition data which were to be predicted, but from separate letter recognition experiments. In addition, the word vocabulary was taken independently from published counts and a limited survey. Thus, no word knowledge parameters were estimated from the word recognition data either. Finally, it should be realized that though the experimental data on which the data are based suited the requirements of an application of the model reasonably well, the number of observations per word and per letter was rather limited. Consequently, results are shown only for all words presented at one eccentric position, since not only individual word predictions are prone to stochastic fluctuations but experimental word responses are so even more. Nevertheless, the present results have interesting implications which will be discussed in the following sections.

\section{Retinal Locations}

In eccentric vision, recognition decreases with eccentricity, but for words the decrease in the right visual field is less than it is in the left. This rightfield advantage has been shown for English words (Mishkin \& Forgays, 1952) and for Dutch words (Bouma, 1973), but not for Hebrew, where, on balance, little left-right difference has been found (Orbach, 1952). It seems, then, that a certain relation exists with the direction of reading. Usually, the right-field advantage is taken to reflect the general language specialization of the left cerebral hemisphere, to which the right visual field at first projects. A relation is then assumed with the advantage of the right ear for speech (Bakker, 1970; Kimura, 1961) and with the localization of speech centers mainly in the left cerebral hemisphere (Geschwind, 1970; Penfield \& Roberts, 1959).

The present model indicates that the right-field advantage for words of three letters is already fully expressed at the level of the constituent letters. Therefore, rather than relating to a general language specialization at levels of encoding more complex than words, these results point to a more efficient coding at a less complex level, perhaps even before the level of letter recognition.

\section{Visual Interference and Letter Recognition}

The most outstanding difference between the present model and the other recognition models discussed is the incorporation of visual interference. The incorporation is implicit; no theory for the specific effects of interference has been developed here. Its effects are taken to be position-specific and are maintained in the model by distinguishing between letters and their positions.

The logogen model does not contain sensory assumptions, but in view of the considerable differences in recognizability resulting from letter position and from word location in the visual field, visual factors might be at least as important as effects of expectancy or word frequency.

The multicomponent model explicitly states independence between feature detectors irrespective of position, though this property has not been tested in the data reported by Rumelhart and Siple (1974). In their experimental conditions, however, the effects of visual interference may have been minimized. The large words, subtending $1.3^{\circ} \times 2.8^{\circ}$ visual angle, made up of capitals were presented foveally for durations of the order of milliseconds. It is a welldocumented fact that the number of elements, for example letters, in a visual display reduces the legibility of each of them. This is especially the case in the parafoveal field and tends to become worse when the elements are closer to each other (Bouma, 1970). Eriksen and Rohrbaugh (1970) found that recognition accuracy decreased progressively when distance between elements was varied from $.78^{\circ}$ to $.08^{\circ}$ visual angle. For the typeface used in the experiments which are discussed here, the average distance is $.05^{\circ}$ visual angle. Townsend, Taylor, and Brown (1971) found severe reduction of visibility of letters in strings of eight with unlimited viewing time.

The effect is also apparent in the time subjects need to decide on the identity of a designated letter between others. Eriksen and his associates propose one interpretation, holding that the other elements in the display interfere with, or slow down, the processing of the target element (Colegate, Hoffman, \& Eriksen, 1973; Eriksen \& Eriksen, 1974; Eriksen \& Hoffman, 1972). Estes (1972) has argued that decreased visual performance which has been interpreted in terms of shifting or focusing of attention can be accounted for in a more coherent way by visual interaction. His interactive channels theory has been formulated quantitatively by Wolford (1975), but not on the level of letter confusions. In this respect, Wolford (1975) presents data reported by Hollingworth and Wolford showing that under 
almost the same conditions as Bouma's (1973), except for a short presentation time of $40 \mathrm{msec}$, interference starts within $1^{\circ}$ from the fixation point. Recently, Eriksen and Schultz (1977) have shown convincingly that it is mainly the elementary properties of the visual system that affect processing rates for stimuli in the visual field. Response times for single letters increased sharply and linearly with eccentricity where visual acuity decreases. Correspondingly, when the stimulus is degraded, response times are even further increased. On the other hand, positional uncertainty does not have any effect. The rate of information extraction is not uniform over the visual field. This rate is then more reduced by the presence of other stimuli, as is the case for words having more letters. This effect is also clear in the whole, partial, or single report paradigm for tachistoscopic recognition. This task is in several respects similar to the letter recognition tasks discussed here, at least when the presentation duration does not exceed $200 \mathrm{msec}$. Such experiments typically employ eight unconnected letters in a row with the fixation point in the middle of the string. The function relating accuracy of report to stimulus position in the string is $\mathrm{W}$-shaped, but there is a strong interaction with order of report or order of cognitive scanning. This causes the normally encountered performance enhancement on the lefthand side (Bryden, 1966; Haber \& Standing, 1969; Merikle, Coltheart, \& Lowe, 1971; Schwantes, 1978; Smith \& Ramunas, 1971). The $W$ shape found is completely in line with the present findings on interference operative in letter recognition in strings. In the middle of the string, near the fixation point, foveal acuity is highest and therefore the middle letters can be better discerned and reported. The outward letters are least affected by lateral interference and are consequently more accurately reported than the more inward ones, even though these are closer to the fovea. Such a sensory effect is expected to hold very generally, it is also apparent when two different words or nonwords are presented on each side of the fixation points, as in the experiments of Krueger (1976). In his experiments, increased response times for Letter Positions 2 and 3 bear witness to reduced sensory information at those positions.

Haber and Standing (1969) could stress their argument for less metacontrast by showing that accuracy of report for the end items dropped substantially when the array was preceded and ended by parentheses. The same type of interference appears in Figures 2 and 4; at greater eccentricities, decreasing acuity and increasing interference make words less recognizable. Initial and final letters are less subject to interference, but it is a curious phenomenon that the letters furthest removed from the fovea are best recognized. The robustness of these sensory effects, the foveal acuity effect and lateral interference effects (Bouma, 1970; Merikle, Coltheart, \& Lowe, 1971), even after report delays of up to $2 \mathrm{sec}$ (Smith \& Ramunas, 1971) seems to leave little room for the effects of control mechanisms (Schwantes, 1978) except for the apparent left-to-right processing when about eight stimuli have to be recalled.

\section{Completion and Redundancy}

Middle letters are much more often correct when they are reported from words than from meaningless strings. As mentioned before, this is thought to be the completion effect: letters which have not been completely perceived may be inferred from knowledge of the word. From a perceptual point of view, word knowledge serves to increase redundancy, which need not be limited to words. One of the basic studies in this field that uses a recognition paradigm is the experiment by Colegate and Eriksen (1972). They varied several forms of redundancy in letter strings (whereby two letters always appeared together in strings of three) and found a higher proportion of correctly reported letters in redundant strings. The particular type of redundancy had only slight effects. Trying to describe the redundancy effect with a simple rule, Colegate and Eriksen (1972) showed that a perceptual independence model could describe the results satisfactorily. In the present context, this means that each letter in the string provides perceptual information which is independent of the information from other letters. This independence condition is the same as that employed in the present model. The stimulus conditions in their experiment resemble those in the word recognition experiments reported here and differ only in minor details. The phenomenon that redundancy contributes most under reduced discriminability conditions, according to results cited by Colegate and Eriksen (1972), is clearly borne out by the data of Figure 4 .

\section{Response Availability}

As has been noted in the foregoing, both predicted correct scores and completion scores were uniformly too low by a small amount. This underestimation is also apparent in the predicted standard deviations of the correct word scores (Table 2). If the correct word scores are scaled in such a way that the underestimation disappears, then the standard deviations are completely in line with the observed deviations. Consequently, erroneous responses are overrepresented in the predictions, and this phenomenon might reflect inadequacies in the assumed accessibility of the responses in the adopted vocabulary. First, the underestimation of predicted correct scores is less with a smaller word vocabulary. In an initial application of the model with a 409-word vocabulary, the predicted correct scores were, on average, 
.035 too low as compared with the .08 for the present 541 word vocabulary as employed here. The immediate effect of an increased number of words in the vocabulary is to increase, generally, the number of possible alternatives for any stimulus word. Consequently, the large sum of their probabilities decreases the probabilities of the correct response by the operation of the constant ration rule. If the underlying model is relevant, subjects would seem to have fewer than 400 responses accessible at any one time. There are several ways of reflecting this in the model while maintaining the vocabulary size. Differential weighting schemes, however, would require free parameters to be introduced which could thus far be avoided. We note here, in passing, that differential accessibility would be somewhat analogous to the application of frequency of occurrence in the predictions. The vocabulary fails on the occasional report of nonsense words. In such a case, the sensory information apparently does not succeed in activating vocabulary entries. As a rule, these strings are pronounceable, suggesting that articulatory readiness could be involved.

A few erroneous responses were reported earlier by the subjects in the recognition trials. Sequential word response bias (Morton, 1969) might be responsible for these repetitions when sensory information is apparently limited. Empirically, one could more safely argue in favor of sequential bias if the visual predictability of the response could be assessed mare reliably. Thus, the recognition model might be employed to study sequential word bias more accurately.

Finally, some words were reported which were not predicted by the model, or predicted to be very improbable. This finding must be expected when the number of observations in the letter recognition study is limited. When only few observations are run, the likelihood that a particular letter confusion, which has a low a priori probability, does not occur at all is rather high. Consequently, no word responses would be predicted containing that confused letter, but they might occasionally be reported by a subject.

\section{Frequency Effects}

Ever since Solomon and Postman (1952), word frequency has been the most extensively studied determinant of word recognition. Apart from its dynamic appeal, the universal interest in frequency stems from its basic simplicity as a single variable factor. It is, therefore, not surprising to find frequency as one of the central issues in the logogen model and in the multicomponent model.

Generally, word frequency is expressed as number of occurrences in printed text and it is assumed to reflect the number of times a subject encounters that word. More frequently occurring words should then be easier to recognize. However, it is difficult to establish which these frequent words are for any given individual, even when the supposed impact of frequency is valid. In the two word-frequency counts that were available to us, the 1937 De la Court count (Linschoten, Note 1) and that of Uit den Bogaart (1975), differences were, of course, to be expected since 40 years separated them, though the kind of material sampled was basically the same. In both counts, the less frequent words, of the order of $10^{-6}$, counted once out of a total of a million, necessarily have a highly unreliable frequency estimate.

Unreliabilities are also apparent from substantial frequency differences between the same words in printed and spoken text (Uit den Boogaart, 1975), even for frequent words. Taken together, these observations seem to indicate that frequency counts inadequatly reflect exposure of words to individual subjects, let alone word availability.

An important question is how large the frequency effect is, as compared to ever-present visual effects as in reading. Under reasonably favorable perceptual conditions, visual effects predominate over those due to frequency, as may be gathered from the data reported by Morton (1968) and by Rumelhart and Siple (1974). Here an attempt has been made to show how much of word recognition behavior can be explained without taking differences in word frequency into account. Even so, the adopted vocabulary represents the basic frequency aspect: the existence of words.

\section{Letter Features vs. Word Features}

It was established around the turn of the century that letter properties alone could not account fully for word recognition, though any notion of redundancy was markedly absent. Neisser (1967) has given a review of these findings, emphasizing the influence of general word shape. The objective features of single letters cannot account fully for general word shape, as they are subject to mutual interference. However, since the amount of interference is dependent on eccentricity, a single word can take different shapes over different positions in the visual field, thereby losing its general word shape. This situation is aggravated in normal text where other words come to occupy nearly all empty spaces and introduce additional interference. In the present model, the letter-based approach has been combined with global aspects by considering the position of letters. Essentially, the model defines a probability distribution over letter strings and some words, the constituent letters of which have been perceived for the stimulus word. The importance of letters in the per- 
ception of words has been emphasized more recently by Thompson and Massaro (1973).

In view of the unclear status of letter features, an explicit feature detection theory was not felt to be necessary for the present application of the model. Two difficulties are connected with a feature theory. First, the choice of features entails an arbitrariness that is difficult to avoid. Neither Gibson's features (1965) nor those of Rumelhart and Siple (1974) have been seriously investigated as to the existence of other possibilities. Next, there is the feature combination problem. Smith (1973), in auditory recognition, and Hubert (1972), in visual recognition, found indications of strong dependence between the hypothesized features. Dependencies of this sort would complicate general recognition theories considerably.

\section{Experimental Limitations}

All letter confusion probabilities were directly taken from the experimental data of letter recognition and are prone to stochastic uncertainty because of the restricted number of letter presentations. In view of the four eccentricities, three letter positions, and the approximately $20 \times 26$ letter confusions, there is no easy solution to this problem (20 letters owing to distributional constraints of letters in words, while subjects may still report up to 26 different letters. For one particular word, there seems indeed to be too little information to obtain accurate predictions. We tried, instead, to reduce the unreliability by averaging over stimuli, and it appears indeed that these averages conform sufficiently well to the observed data.

An unavoidable consequence of seeing the stimulus only once is that the responses of the subjects represent only a sample of all possible reponses theoretically obtainable. This is a problem for any word recognition model, though not all responses would appear to be interesting from a perceptual standpoint. In order to avoid some of the experimental limitations, new experiments have been carried out involving only words of three letters, where the number of presentations of both words and letters were sizably increased. A preliminary account of these experiments is in preparation.

\section{Conclusion}

The results of the test of the word recognition model presented here showed that the word recognition in the earlier experiment by Bouma (1973) can be satisfactorily predicted from letter recognition data and word knowledge. The results suggest, too, that the features of words, for example, global word shape, responsible for word recognition might be satisfactorily described in terms of the letters in their position. Within the present experimental conditions, these results are valid for parafoveal recognition only, since foveally presented words are generally perfectly seen in 100 -msec presentations. The method can, however, easily be extended, for instance by shortening the presentation time for foveal presentation in order to degrade stimulus quality.

The simplicity of the present model is attained at the expense of a large number of fixed letter recognition parameters, estimated in separate experiments. This is advantageous with respect to the number of assumptions to be made, which is minimized in this scheme. Any further development would eventually be directed at greater theoretical power in the description of letter perception. In this respect, the independence of letter perception, which is in line with the results of Colegate and Eriksen (1972), is a useful and simplifying condition.

Finally, a better insight is needed into the subject's individual vocabulary and its instantaneous accessibility. The word list employed for the model is at best a very rough approximation as regards content and seems additionally to overestimate the number of accessible words. A study of word knowledge has been made in which a lexical decision task was used, the results of which indicate that words, indeed, vary greatly in accessibility (Bouwhuis, in press).

The results of the latter study also indicate that subjects share their word knowledge in the sense that they know the same words well and they all have difficulties with little-known words. In addition, in support of the present model, word knowledge appeared to be largely independent of word frequency.

\section{REFERENCE NOTE}

1. Linschoten, J. De la Court's frequentietelling van Nederlandse woorden (Report No. 6301). Utrecht: Psychology Laboratory, 1963.

\section{REFERENCES}

BAKKER, D. J. Ear asymmetry with monaural stimulation: Relations to lateral dominance and lateral awareness. Neuropsychologia, 1970, 8, 103-117.

Bouma, H. Interaction effects in parafoveal letter recognition. Nature, 1970, 226, 177-178.

Bouma, H. Visual recognition of isolated lower-case letters. Vision Research, 1971, 11, 459-474.

Bouma, H. Visual interference in the parafoveal recognition of initial and final letters of words. Vision Research, 1973, 13, $767-782$.

Bouwhuis, D. G. Word knowledge and letter recognition as determinants of word recognition. In P. A. Kolers. M. E. Wrolstadt, $\&$ H. Bouma (Eds.), The processing of visible language 1 . New York: Plenum, in press.

Bryden, M. P. Accuracy and order of report in tachistoscopic recognition. Canadian Journal of Psychology, 1966, 20, 262-272.

Clarke, F. R. Constant-ratio rule for confusion matrices in speech communication. Journal of the A coustical Society of America, 1957, 29, 715-720.

Colegate, R. L.. \& Eriksen, C. W. Form of redundancy as a determinant of tachistoscopic word recognition. Perception \& Psychophysics, 1972, 12, 477-481. 
Colegate, R. L., Hoffman, J. E., \& Eriksen, C. W. Selective encoding from multielement visual displays. Perception \& Psychophysics, 1973, 14, 217-224.

ERIKSEN, B. A., \& Eriksen, C. W. Effects of noise letters upon the identification of a target letter in a nonsearch task. Perception \& Psychophysics, 1974, 16, 143-149.

ERIKSEN, C. W., \& Hofrman, J. E. Temporal and spatial characteristics of selective encoding from visual displays. Perception \& Psychophysics, 1972, 12, 201-204.

Eriksen, C. W., \& Rohrbaugh, J. W. Some factors determining efficiency of selective attention. American Joumal of Psychology, 1970, 83, 330-342.

ERIKSEN, C. W., \& Schultz, D. W. Retinal locus and acuity in visual information processing. Bulletin of the Psychonomic Society, 1977, 9, 81-84.

ESTES, W. K. Interactions of signal and background variables in visual processing. Perception \& Psychophysics, 1972, 12, 278-286.

GESCHWIND, N. The organization of language and the brain. Science, 1970, 170, 940-944.

Gibson, E. J. Learning to read. Science, 1965, 148, 1066-1072.

Haber, R. N., \& Standing, L. Location of errors with a poststimulus indicator. Psychonomic Science, 1969, 17, 345-346.

Hubert, L. A statistical method for investigating the perceptual confusions among geometric configurations. Journal of Mathematical Psychology, 1972, 9, 389-403.

KIMURA, D. Cerebral dominance and the perception of verbal stimuli. Canadian Journal of Psychology, 1961, 15, 166-171.

KRUEGER, L. E. Evidence for directional scanning with the order of report factor excluded. Canadian Journal of Psychology, 1976, 30, 9-14.

LUCE, R. D. Individual choice behavior. New York: Wiley, 1959.

Merikie, P. M., Coltheart, M., \& Lowe. D. G. On the selective effects of a patterned masking stimulus. Canadian Journal of Psychology, 1971, 25, 264-279.

Mishin, M., \& ForgaYs, D. G. Word recognition as a function of retinal locus. Journal of Experimental Psychology, 1952, 43, 43-48.

MorTon, J. Interaction of information in word recognition. Psychological Review, 1969, 76, 165-178.
NeIsser, U. Cognitive psychology. New York: Appleton-CenturyCrofts, 1967.

ORBACH, J. Retinal locus as a factor in the recognition of visually perceived words. American Journal of Psychology, 1952, 65, 555-562.

Penfield, W., \& Roberts, L. Speech and brain mechanisms. New Jersey: Princeton University Press, 1959.

Rumelhart, D. E., \& Siple, P. Process of recognizing tachistoscopically presented words. Psychological Review, 1974, 81, 99-118.

SCHWANTES, F. Stimulus position functions in tachistoscopic identification tasks: Scanning, rehearsal, and order of report. Perception \& Psychophysics, 1978, 23, 219-226.

Solomon, R. L., \& Postman, L. Frequency of usage as a determinant of recognition thresholds for words. Journal of Experimental Psychology, 1952, 43, 195-201.

Smith, M. C., \& Ramunas, S. Elimination of visual field effects by use of a single report technique. Journal of Experimental Psychology, 1971, 87, 23-28.

SMITH, P. T. Feature testing models and their application to perception and memory for speech. Quarterly Journal of Experimental Psychology, 1973, 25, 511-534.

Thompson, M. C., \& Massaro, D. W. Visual information and redundancy in reading. Journal of Experimental Psychology, 1973, 98, 49-54.

Townsend, J. T., TAYloR, S. G., \& BRown, D. R. Lateral masking for letters with unlimited viewing time. Perception \& Psychophysics, 1971, 10, 375-378.

UIT DEN BOOGAART, P. C. Woordfrequenties in geschreven en gesproken Nederlands. Utrecht: Oosthoek/Scheltema en Holkema, 1975.

W HEFLER, D. Processes in word recognition. Cognitive Psychology, $1970,1,59-85$

Wolford, G. Perturbation model for letter identification. Psychological Review, 1975, 82, 184-199.

WoOdworTH, R. S. Experimental psychology. New York: Henry Holt, 1938.

(Received for publication August 22, 1978; accepted September 26, 1978.) 